\title{
Unerwünschte Arzneimittelwirkungen
}

\section{QT-Verlängerungen und Torsade-de-Pointes-Arrhythmien}

\section{S. Haffner ${ }^{1}$ \\ H. Lapp ${ }^{2}$}

P. A. Thürmann ${ }^{1}$

\section{Drug induced QT-prolongation and Torsade-de-Pointes arrhythmia}

Repolarisationsstörungen wie QT-Intervall-Verlängerung oder morphologische $\mathrm{T}(\mathrm{U}-)$-Wellenveränderungen und die damit in Zusammenhang stehende Auslösung von potenziell fatalen Torsade-de-Pointes(TdP)-Tachykardien ist eine derzeit vieldiskutierte kardiale Nebenwirkung von Arzneimitteln. Während diese bei rhythmuswirksamen kardialen Medikamenten eher erwartet und bedacht werden, bleibt diese Gefahr bei der Verschreibung nicht-kardialer Arzneimittel oft unberücksichtigt (Tab.1). Immerhin führte das proarrhythmische Potential zur Marktrücknahme einiger Präparate, z.B. Prenylamin, Terodilin, Sertindol, Astemizol und Cisaprid. Bei Terfenadin, Budipin, Pimozid und Halofantrin wurden Verschreibungsauflagen bzw. Warnhinweise ausgesprochen. Daran ist zu erkennen, dass erhebliche Schwierigkeiten bei der Erkennung von proarrhythmischen Effekten in der präklinischen und klinischen Arzneimittel-Testung bestehen. Die Wachsamkeit bei der Anwendung nach der Zulassung muss deshalb umso größer sein. Die Inzidenz von TdP ist zwar bei den meisten hier erwähnten Substanzen gering, kann aber bei einigen Antiarrhythmika wie Chinidin und Sotalol bis zu 5\% der Exponierten betreffen (5). Die Brisanz dieser unerwünschten Arzneimittelwirkung (UAW) ergibt sich durch die potenzielle Lebensbedrohung (Synkopen, plötzlicher Herztod), die subjektiv geringe Symptomatik und die Neigung, kardial nicht wirksame Medikamente auch nicht mit Rhythmusstörungen in Verbindung zu bringen. Die proarrhythmogenen Effekte können durch pharmakodynamische und -kinetische Arzneimittelinteraktionen potenziert werden. Zudem erfordert die genauere Beurteilung kardialer Repolarisationsstörungen kardiologische Erfahrung.

kurzgefasst: QT-Verlängerung und eine sich daraus entwickelnde Torsade-de-Pointes-Tachykardie sind mögliche lebensbedrohliche unerwünschte Arzneimittelwirkungen einiger häufig benutzter Substanzgruppen.

\section{QT-Verlängerung und TdP-Gefahr}

Das QT-Intervall repräsentiert die Summe der Aktionspotentialdauern der einzelnen ventrikulären Kardiomyozyten. Seine Verlängerung bei normaler QRS-Morphologie wird typischerweise durch zelluläre Repolarisationsstörungen hervorgerufen. Während die Depolarisation hauptsächlich durch $\mathrm{Na}^{+}-$und (vor allem im Sinus- und AV-Knoten) $\mathrm{Ca}^{2+}$-Kanäle bedingt ist, spielt bei der Plateau- und Repolarisationsphase hauptsächlich ein langsam abnehmender $\mathrm{Ca}^{2+}$-Einstrom und ein zunehmender $\mathrm{K}^{+}$-Ausstrom eine Rolle. Verschiedene Medikamente verändern den Ablauf dieser empfindlichen ausbalancierten Mechanismen durch Veränderung von Leitfähigkeiten der Ionenkanäle. Entscheidend ist hier eine medikamentöse Verlängerung des Aktionspotentials entweder durch Behinderung des repolarisierenden $\mathrm{K}^{+}$-Ausstroms oder durch Verlängerung des $\mathrm{Na}^{+}$- oder $\mathrm{Ca}^{2+}$-Einstroms. Vermutlich kommt es durch unterschiedliche Verteilung der beeinträchtigten Kanäle innerhalb des Myokards zu inhomogenen Erregungszuständen, die sich im EKG als Nachpotentiale, Änderung der T(U)-Wellen-Morphologie und zunehmende QT-Dispersion äußern und in der Summe QT-verlängernd wirken. Diese Vorgänge prädisponieren zu frühen ventrikulären Extrasystolen mit der Gefahr der Auslösung von TdP-Tachykardien (7). Somit ist neben der veränderten $\mathrm{T}(\mathrm{U})$-Wellen-Morphologie hauptsächlich eine Verlängerung des QT-Intervalls als Surrogatparameter für die Gefahr der Auslösung von TdP-Arrhythmien geeignet. Es zeigt sich allerdings, dass Länge des QT-Intervalls und TdP-Neigung nicht immer korrelieren müssen: Bekannte Ausnahmen sind Amiodaron (5) und Bepridil (ein Verapamil-artiger Ca-Antagonist), die zwar einen ausgeprägten Effekt auf das QT-Intervall haben, jedoch weniger häufig zur TdP zu führen scheinen, während Klasse Ia-Antiarrhythmika ein hohes TdP-Risiko bei einer vergleichsweise geringen QT-Intervallverlängerung aufweisen.

${ }^{1}$ Philipp Klee-Institut für Klinische Pharmakologie, Klinikum Wuppertal GmbH, Lehrstuhl für Klinische Pharmakologie der Universität Witten/Herdecke

2 Medizinische Klinik 3, Herzzentrum Klinikum Wuppertal GmbH,

Lehrstuhl für Kardiologie der Universität Witten/Herdecke

Korrespondenz

Prof. Dr. med. Petra A. Thürmann · Philipp Klee-Institut für Klinische Pharmakologie

Klinikum Wuppertal GmbH · Heusnerstraße 40 - 42283 Wuppertal · Tel.: 0202/8961851

· Fax: 0202/8961852 ·E-Mail: petra.thuermann@klinikum-wuppertal.de

eingereicht: 21.1 .2002 - alkzeptiert: 17.4 .2002 
kurzgefasst: Die Verlängerung des QT-Intervalls und Änderungen der $T(U)$-Wellen-Morphologie sind klinische Marker zur Abschätzung der Gefahr von Torsade-dePointes-Tachykardien und malignen Arrhythmien.

\section{Prädisponierende QT-Intervall-verlängernde Faktoren}

Die Ausgangslänge des QT-Intervalls, auf die sich Medikamenteneffekte aufsummieren, wird von verschiedenen physiologischen Faktoren beeinflusst. Zudem bestehen zahlreiche erbliche oder erworbene Veränderungen der in den Repolarisationsmechanismus involvierten Ionenkanäle. Ausgehend von diesen prädisponierenden Faktoren wird die Toleranz gegenüber QT-verlängernden Zusatzfaktoren als „Repolarisationsreserve“ bezeichnet.

Der wichtigste physiologische Faktor ist die Herzfrequenz: Bei Bradykardie verlängert sich das QT-Intervall, bei Tachykardie muss bei einer Beurteilung eine Verkürzung berücksichtigt werden (Korrekturverfahren s.u.). Daneben bestehen auch Einflüsse durch autonomes Nervensystem und Elektrolythaushalt (vagusinduzierte Bradykardie sowie Hypokaliämie, Hypocalcämie und Hypomagnesiämie). Ebenso nimmt auch mit zunehmendem Alter die QT-Zeit und -Variabilität zu $(8,11)$. Hormonelle Einflüsse spielen eine Rolle $(3,11,13)$ : Frauen weisen, in Abhängigkeit von ihrem Menstruationszyklus, im Vergleich zu Männern verlängerte QT-Zeiten und ein empfindlicheres Ansprechen auf proarrhythmische Eigenschaften vieler Medikamente auf (Androgen- und Progesteron-Schutzwirkung bzw. Östrogen-Prädisponierung). Tab.2 listet weitere prädisponierende Faktoren auf, die mit QT-Verlängerung und TdP-Neigung einhergehen, darunter vor allem Erkrankungen, die mit kardialen elektrischen Erregungsinhomogenitäten und mit Bradykardieneigung einhergehen. Weiterhin soll hier noch auf die vielfältigen Formen der kongenitalen Long-QT-Syndrome (CLQTS), klinisch in ihrer ausgeprägten Form unter Romano-Ward- und (in Kombination mit Taubheit) Jervell-Lange-Nielsen-Syndrom geführt, hingewiesen werden, die nicht immer klinisch auffällig sein müssen (12). Molekularbiologische Arbeiten konnten zahlreiche Mutationen identifizieren, die teils nur zu geringfügigen Funktionseinschränkungen der Kanalproteine führen ohne das QT-Intervall deutlich zu verlängern. Diese können unter dem zusätzlichen Einfluss anderer Risikofaktoren (Tab.1, Herzinfarkt, Herzhypertrophie, außergewöhnliche vegetative Belastungen) nicht mehr kompensiert werden und können so zur Arrhythmie führen (10,15). Die Inzidenz aller Formen des CLQTS wird auf 1:5000 geschätzt (15).

kurzgefasst: Exogene Faktoren sowie erbliche und erworbene krankhafte Veränderungen können durch Schmälerung der „Repolarisationsreserve“ zu Torsade-de-Pointes-Tachykardien prädisponieren.

\section{QT-Prolongation und TdP-Tachykardien durch Medikamente}

In Tab.1 sind Substanzen aufgeführt, über die Berichte von TdP oder QT-Verlängerung vorliegen. Wie oben angeführt, kann eine medikamentös induzierte QT-Verlängerung zu einer TdP führen. Die Zahl verdächtigter Medikamente steigt aufgrund der zunehmenden Aktualität der Thematik ständig an $(2,16)$.
Tab.1 Arzneimittelgruppen, die zur Verlängerung des QT-Intervalls und Auslösung von Torsade-de-Pointes-Tachykardien führen können (in Anlehnung an 4, 6 und 15).

\begin{tabular}{|c|c|}
\hline Arzneimittelgruppe & Wirkstoffe \\
\hline \multicolumn{2}{|l|}{ Antiarrhythmika } \\
\hline Klasse la: & Chinidin, Procainamid, Disopyramid, Ajmalin \\
\hline Klasse lb: & Mexiletin \\
\hline Klasse lc: & $\begin{array}{l}\text { Flecainid, Propafenon, (eher durch QRS -Verbrei- } \\
\text { terung proarrhythmisch: Lorcainid und Tocainid) }\end{array}$ \\
\hline Klasse III: & Amiodaron, Sotalol, Ibutilid, Dofetilid \\
\hline andere: & Adenosin \\
\hline $\begin{array}{l}\text { Calcium- } \\
\text { Antagonisten: }\end{array}$ & $\begin{array}{l}\text { Isradipin, Nicardipin, in D nicht auf dem Markt: } \\
\text { Bepridil, möglicherweise QT-Verlängerung: Vera- } \\
\text { pamil; nicht mehr auf dem Markt: Mibefradil }\end{array}$ \\
\hline Neuroleptika & $\begin{array}{l}\text { Thioridazin, Chlorpromazin, Haloperidol, Mesorida- } \\
\text { zin, Pimozid, Quetiapin, Risperidon, Ziprasidon }\end{array}$ \\
\hline Antidepressiva & $\begin{array}{l}\text { Amitriptylin, Desipramin, Imipramin, Doxepin, Fluo- } \\
\text { xetin, Paroxetin, Venlafaxin, Sertralin, Citalopram, } \\
\text { Lithium, unklarer Mechanismus: Trazodon }\end{array}$ \\
\hline $\begin{array}{l}\text { Dopaminerge und } \\
\text { serotoninerge } \\
\text { Wirkstoffe: }\end{array}$ & $\begin{array}{l}\text { Domperidon, (weniger Metoclopramid, hier sind } \\
\text { eher supraventrikulär proarrhythmische/ sinusblo- } \\
\text { ckierende Wirkungen beschrieben), Dolasetron, } \\
\text { Zolmitriptan, Sumatriptan, Naratriptan, vom } \\
\text { Markt genommen: Cisaprid }\end{array}$ \\
\hline $\begin{array}{l}\text { Andere zentral } \\
\text { wirksame Stoffe: }\end{array}$ & $\begin{array}{l}\text { Budipin, Droperidol, Chloralhydrat, Felbamat, } \\
\text { Fosphenytoin, Cocain, Levomethadon }\end{array}$ \\
\hline Antihistaminika & $\begin{array}{l}\text { Terfenadin, weniger auch: Azelastin, Loratadin } \\
\text { und Cetirizin, vom Markt genommen: Astemizol }\end{array}$ \\
\hline \multicolumn{2}{|l|}{ Antiinfektiva: } \\
\hline Makrolide: & $\begin{array}{l}\text { Clarithromycin, Erythromycin, Roxithromycin, } \\
\text { Azithromycin, Spiramycin }\end{array}$ \\
\hline Gyrasehemmer: & $\begin{array}{l}\text { Sparfloxacin, Grepafloxacin, Moxifloxacin, } \\
\text { Gatifloxacin, Levofloxacin }\end{array}$ \\
\hline Andere Antibiotika: & Pentamidin, Clindamycin, fraglich: Cotrimoxazol \\
\hline Antimykotika: & $\begin{array}{l}\text { Fluconazol, (Ketoconazol, Miconazol und Itracona- } \\
\text { zol nur indirekt proarrhythmisch durch Metabolis- } \\
\text { mushemmung von anderen proarrhythmischen } \\
\text { CYP 3A4-metabolisierten Substanzen) }\end{array}$ \\
\hline Virustatika: & Foscarnet, Amantadin \\
\hline Antimalaria-Mittel: & $\begin{array}{l}\text { Halofantrin, Chinin, möglicherweise auch: } \\
\text { Chloroquin und Mefloquin }\end{array}$ \\
\hline \multicolumn{2}{|l|}{ Andere Arzneimittel } \\
\hline Sympathomimetika: & $\begin{array}{l}\text { in höheren Dosen oder bei prädisponierten Perso- } \\
\text { nen: } \beta_{2} \text {-Mimetika (Fenoterol,Formoterol, Salmete- } \\
\text { rol), Catecholamine, Amphetaminabkömmlinge } \\
\text { (z.B. Sibutramin), Vasokonstriktoren wie Ephedrin } \\
\text { und Etilephrin }\end{array}$ \\
\hline $\begin{array}{l}\text { Immunreaktions- } \\
\text { hemmer: }\end{array}$ & Tacrolimus \\
\hline $\begin{array}{l}\text { Zytostatisch wirkende } \\
\text { Medikamente: }\end{array}$ & Arsentrioxid, Tamoxifen \\
\hline Verschiedene: & Indapamid, Moexipril, Probucol, Tizanidin, Octreotid \\
\hline
\end{tabular}

Prinzipiell können Arzneimittel entweder die QT-Zeit selbst verlängern, z.B. durch Angriff an den Kaliumkanälen $\mathrm{I}_{\mathrm{Kr}}$ und/oder $\mathrm{I}_{\mathrm{Ks}}$, oder sie können Begleitfaktoren beeinflussen (z.B. Elektrolytkonzentrationen), außerdem sind pharmakokinetische/-dynamische Interaktionen zu berücksichtigen (2). Eine ausgesprochene Plasmakonzentrationsabhängigkeit für die QT-Prolongation konnte zwar nicht generell etabliert werden, doch muss trotzdem von einem erhöhten Risiko bei erhöhten Blutspiegeln z.B. durch interak- 
Tab.2 Zustände, die mit einer Verlängerung des QT-Intervalls einhergehen und zu arzneimittelinduzierter QT-Verlängerung prädisponieren (nach 5).

$\begin{aligned} & \text { Kongenitale Long-QT-Interval-Syndrome (z.B. Romano-Ward- und Jervell- } \\ & \text { Lange-Nielsen-Syndrom) }\end{aligned}$
Elektrolytstörungen (Hypokaliämie, Hypocalcämie, Hypomagnesiämie)
KHK, Kardiomyopathie, Hypertonie, Bradykardie
Hypoglykämie, Diabetes mellitus, Hypothyreoidismus, Hypophyseninsuffizienz
ZNS-Alterationen (Apoplex, Trauma, Tumor, Infektion)
Adipositas
Alkoholismus und Cocain-Missbrauch
Bestimmte Proteindiäten
Weibliches Geschlecht (auch zyklusabhängig)
höheres Alter
Nahrungsaufnahme, Schlaf

tive Metabolismushemmung (v.a. bei Cytochrom P450-3A4- und -2D6-abhängigem Metabolismus), besonders bei Vorliegen eines „poor-metabolizer“-Status oder hepatischer Insuffizienz, ausgegangen werden. Bedeutsam in pharmakokinetischer Hinsicht sind auch das Verteilungsvolumen (evtl. wesentlich höhere kardiale Gewebespiegel als Plasmaspiegel), die Art der Metaboliten (QTwirksame Metaboliten) sowie die renale Funktion. Auf pharmakodynamischer Ebene wird die Gefahr potenziert durch gleichzeitige Verabreichung eines anderen (auch indirekt) QT-verlängernden Stoffes. Bezüglich Interaktionen müssen neben den MakrolidAntibiotika und den Azol-Antimykotika, die sowohl den CYP 3A4Metabolismus hemmen als auch direkt QT-Intervall-verlängernd wirken, auch die Substanzgruppen der SSRI, der HIV-ProteaseHemmer und der Calcium-Antagonisten sowie Grapefruit-Saft bedacht werden, die allesamt CYP 3A4-Hemmer sind $(2,4)$.

\section{kurzgefasst: Proarrhythmiegefahr durch QT-Verlänge- rung sind bei Vertretern folgender Substanzgruppen zu beachten: Antiarrhythmika, Psychopharmaka, Antiin- fektiva, Antihistaminika, dopaminerge und serotoniner- ge Substanzen. Oft zeigt sich diese fatale Eigenschaft erst nach Kombination mit interagierenden Substanzen.}

\section{Problematik der QT-Intervall-Bestimmung}

Zum einen ist hier die hohe intraindividuelle Variabilität der QT-Dauer zu berücksichtigen, die nach Arbeiten von Pratt et al. (9) bei Gesunden über $50 \mathrm{~ms}$, bei kardial Erkrankten sogar noch höher liegen kann. Klinisch wird daher erst ab einer individuellen Verlängerung des QT-Intervall von über 40-60 ms oder einer absoluten QT-Dauer von $>500 \mathrm{~ms}$ von einem bedrohlichen Effekt ausgegangen. Als eindeutig pathologisch gilt eine QTc-Zeit (s.u.) bei Männern ab 450 ms, bei Frauen ab $470 \mathrm{~ms}(7,9)$.

Durch verschiedene Korrekturverfahren wird versucht, eine Vergleichbarkeit der Intervalle unabhängig von der Herzfrequenz herzustellen. Hierzu wird zumeist die logarithmische Bazett-Formel: $\mathrm{QTc}=\mathrm{QT} \times \mathrm{RR}^{-1 / 2}$ herangezogen, die jedoch bei hohen Frequenzen das QT-Intervall zu lang, bei niedrigen entsprechend zu kurz einschätzt. Dies führt zur Unterschätzung der Arrhythmiegefahr in bradykarden Situationen, die schon als solche einen Risikofaktor darstellen. Als bessere Alternative bieten sich daher neuere Korrekturverfahren an, die - anhand wesentlich größerer Bevölkerungskollektive entwickelt - einen eher linearen Zusammenhang mit der Herzfrequenz annehmen („QTIc“ =QT+1,54 (1-RR)) (14) bzw. autonome/ pathologische Einflussfaktoren besser berücksichtigen (1).

Ein weiterer Unsicherheitsfaktor besteht im Ablesen des QT-Intervalls aus dem EKG-Streifen (5). Mitunter stellen die genaue Abgrenzung der T-Welle sowie Fragen nach Berücksichtigung pathologischer T-Wellen-Morphologien und U-Wellen ein Problem dar, das im Einzelfall fachärztliche Abklärung erfordern kann.

kurzgefasst: Bei der Bestimmung des QT-Intervalls muss berücksichtigt werden, dass es sich nicht um einen statischen Wert handelt, sondern dass es - auch intraindividuell - beträchtlichen Schwankungen durch physiologische und pathologische Einflüsse unterliegt.

Fazit

Nicht nur bei der Verordnung von Antiarrhythmika, sondern insbesondere auch bei vielen Antiinfektiva, Antipsychotika, Antihistaminika sowie dopaminergen, serotoninergen und catecholaminergen Substanzen ist mit der Möglichkeit proarrhythmischer Nebenwirkungen zu rechnen (s. Tab.1). Dies gilt um so mehr bei gleichzeitiger Anwendung von metabolisch interagierenden Substanzen (CYP 3A4- und 2D6-Abbauweg) und bei Vorliegen erblicher Disposition oder kardialer Vorerkrankungen. Trotz gewisser Einschränkungen sind die Verlängerung des QTIntervalls und Änderungen der T(U-) Wellen-Morphologie gute Surrogatmarker zur Abschätzung der Proarrhythmiegefahr.

\section{Literatur}

Davey P. A new physiological method for heart rate correction of the QT interval. Heart 1999; 82: 183-187

2 Dhein S. Arzneimittel-induzierte OT-Verlängerung und Torsade de PointesArrhythmien. Dtsch med Wochenschr 2000; 125: 703-708

3 Drici MD, Clement N. Is gender a risk faktor for adverse drug reactions? The example of drug-induced long QT syndrome. Drug Saf 2001; 24: 575-585

4 Springuel P. Drugs causing prolongation of QT interval and torsade de pointes. Can Adv Drug React Newsl 1998; 8: 1-3

5 Malik M, Camm AJ. Evaluation of drug-induced QT interval prolongation. Implications for drug approval and labelling. Drug Saf 2001; 24: 323-351

${ }^{6}$ Micromedex® Medizinisches Informationssystem, 2001; 110

7 Moss AJ. The QT interval and torsade de pointes. Drug Saf 1999; 21 (Suppl 1); $5-10$

8 Piccirillo G, Cacciafesta $M$, Lionetti $M$ et al. Influence of age, the autonomic nervous system and anxiety on QT-interval variability. Clin Sci (Lond) 2001; 101: 429-438

Pratt CM, Ruberg S, Morganroth J et al. Dose-response relation between terfenadine (Seldane) and the QTc interval on the scalar electrocardiogram: distinguishing a drug effect from spontaneous variability. Am Heart J 1996; 131: 472-480

10 Priori SG, Napolitano C, Schwartz PJ. Low penetrance in the long-QT syndrome: clinical impact Circulation 1999: 99: 529-533

11 Rautaharju PM, Zhou SH, Wong S et al. Sex differences in the evolution of the electrocardiographic QT interval with age. Can J Cardiol 1992; 8: 690-695

12 Roden DM, Lazzara R, Rosen M et al. Multiple mechanisms in the long-QT syndrome. Current knowledge, gaps, and future directions. Circulation 1996: 94: 1996-2012

13 Rodriguez I, Kilborn MJ, Liu X-K, Pezzullo JC, Woosley RL. Drug-induced QT prolongation in women during the menstrual cycle. JAMA 2001; 285: 1322-1326

${ }^{14}$ Sagie A, Larson MG, Goldeberg RJ et al. An improved method for adjusting the QT interval for heart rate (the Framingham Heart Study). Am J Cardiol 992; 70: 797-801

15 Vincent MG. SADS Foundation, Salt Lake City, http://www.sads.org/ LQTS.html,

${ }^{16}$ Woosley RL. Drugs that prolong the QT interval and/or induce torsade de pointes. http://www.georgetowncert.org, updated Oct 2001 